\title{
Bow-tie Antenna for Terahertz Resonant Tunnelling Diode Based Oscillators on High Dielectric Constant Substrate
}

\author{
Khalid Hamed Alharbi, Afesomeh Ofiare, Monageng Kgwadi, Ata Khalid, and Edward Wasige \\ High Frequency Electronics Group, Division of Electronics and Nanoscale Engineering, School of Engineering \\ University of Glasgow, Oakfield Avenue, G12 8LP Glasgow, Scotland, UK \\ Email:k.alharbi.1@ research.gla.ac.uk; edward.wasige@glasgow.ac.uk
}

\begin{abstract}
In this paper, a broadband bow-tie slot antenna is presented. The coplanar waveguide (CPW) fed antenna is fabricated on an InP substrate for same chip integration with the promising resonant tunnelling diode (RTD) terahertz (THz) oscillator which has the capability of room temperature operation and with relative high power. The antenna exhibits a very wide bandwidth (return loss $\mathrm{S11}<-\mathbf{- 1 0} \mathrm{dB}$ ) around the design frequency $(300 \mathrm{GHz})$. However, the radiation pattern is degraded because of the large dielectric constant of the InP substrate. A technique to overcome this problem employing a reflector ground plane underneath a thin substrate of low dielectric constant is presented. Initial simulation results of this technique are reported and experimental validation at lower frequency $(17 \mathrm{GHz})$ shows the feasibility of the concept.
\end{abstract}

Keywords-Terahertz, Technology, Bow-tie Slot Antenna, Resonant Tunnelling Diode, Broadband Antenna

\section{INTRODUCTION}

Terahertz $(\mathrm{THz})$ radiation, whose frequency range lies between millimetrewaves and infrared light in the electromagnetic spectrum has many potential applications in different scientific fields such as medical diagnostics, security imaging, and wireless communication [1-3]. Resonant tunnelling diode (RTD) based $\mathrm{THz}$ oscillators in integrated circuit (IC) form have recently been shown to be the technology in which many of these applications could be implemented [4], [5]. They operate at room temperature and have the potential to produce output power of around $1 \mathrm{~mW}$ at these frequencies [6].

An efficient antenna is a key element to couple the $\mathrm{THz}$ radiation into free space. A high gain and directive antenna is preferable in order to radiate most of the RF power into specific direction and, as a consequence, offer larger coverage distance. RTD THz oscillators employing integrated slot antennas [4] and planar horn antennas [7] have been demonstrated. The slot antenna is a resonant antenna with infinity input impedance at the centre of the slot and zero at the edges. For antenna dimensions of a few microns (at $\mathrm{THz}$ frequencies), proper impedance matching for this antenna is difficult. The planar horn antenna, on the other hand, radiates the signal energy along the substrate, whilst radiation perpendicular to the substrate would be more desirable [8]. An elevated patch antenna was proposed to suppress the substrate effects, however, the bandwidth still narrow [9].

This paper describes a bow-tie slot antenna designed for operation at $300 \mathrm{GHz}$. This antenna has a controlled input impedance of $50 \Omega$. The bow-tie slot antenna is also compatible with the novel power combining RTD oscillator circuits being developed in the group [10]. Most of the radiation in the present design is directed into the substrate due to the high dielectric constant of the Indium Phosphide (InP) substrate [11]. A common solution to this problem has been to use a hemispherical lens on the backside of the substrate to collect the RF power and increase the antenna gain [12-18]. Instead of employing such bulky lenses, a promising alternate realisation to have most of the energy radiated away from the substrate is presented in this paper. It employs a reflector ground plane underneath a thin substrate of low dielectric constant which can be spun on the top of the high dielectric substrate. Initial simulation results of the proposed technique at frequencies around $300 \mathrm{GHz}$ (in addition to experimental validation at lower frequency of $17 \mathrm{GHz}$ ) are introduced in this paper. The frequency of $300 \mathrm{GHz}$ has been suggested for applications such as short-link and medium-link wireless communication systems. This is mainly due to the fact that it is unregulated and in moderate atmospheric attenuation band [19].

\section{BOW-TIE ANTENNA ON HIGH DIELECTRIC CONSTANT} SUBSTRATE

\section{A. Antenna Design}

A bow-tie antenna is a simple version of planar slot antennas which can offer large bandwidth. A simple CPWfed bow-tie antenna adopted in this work was proposed in [20]. Figure 1 shows the geometry of the adopted design. For a desired operating frequency, the dimensions $a, b, L$, and $W$ are calculated as follows:

$$
\begin{array}{r}
a=1.6 \lambda_{o} / \sqrt{\epsilon_{r}} \\
b=0.5 \lambda_{o} / \sqrt{\epsilon_{r}} \\
L=a / 0.85 \\
W=b / 0.45
\end{array}
$$

The antenna was designed to operate at $300 \mathrm{GHz}$ on a semiinsulating Indium Phosphide (InP) substrate with dielectric constant of $\epsilon_{r}=12.56$. Thus, the dimensions $a, b, L$, and $W$ are $451.5 \mu \mathrm{m}, 141 \mu \mathrm{m}, 531 \mu \mathrm{m}$, and $313.5 \mu \mathrm{m}$, respectively. The CPW line dimensions $(\mathrm{g}=20 \mu \mathrm{m}$ and $\mathrm{s}=13.8 \mu \mathrm{m})$ were calculated to give a $50 \Omega$ characteristic impedance. The conducting element is made of $0.4 \mu \mathrm{m}$ thick gold and patterned on the top of the substrate. The antenna was simulated in HFSS 


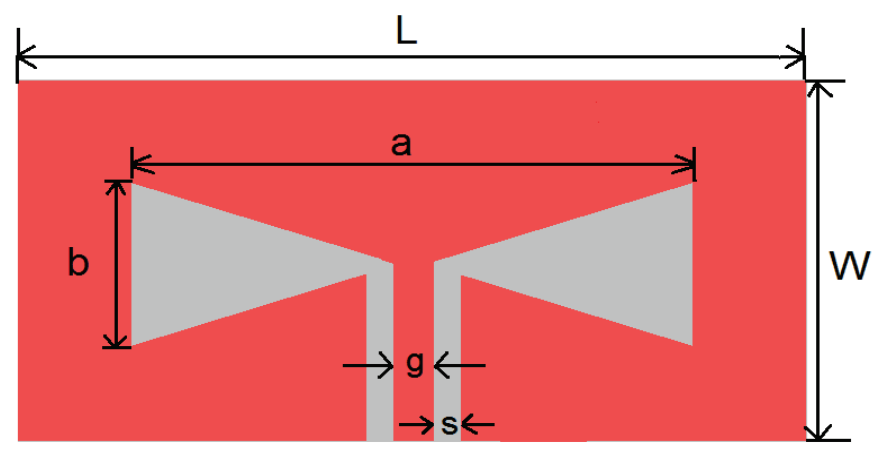

Fig. 1. The geometry of the adopted bow-tie antenna [20]

simulation package which employs finite element method to solve Maxwell's equations. The antenna was fed by a waveport (assigned to a rectangular patch facing the substrate side and the edges of the CPW metals).

\section{B. Antenna Fabrication}

The antenna was fabricated using electron beam lithography process. Electron beam resist PMMA was used. The spun PMMA on the InP sample was baked for 3 minutes at $160^{\circ} \mathrm{C}$, followed by e-beam pattern writing using Vestec VB6 beam writer. After development in 1:1 IPA:MIKB, Ti/Au $(20 \mathrm{~nm} / 400 \mathrm{~nm})$ metal scheme was deposited using an electron beam evaporator followed by lift-off in acetone. Figure 2 is a micrograph of one of the fabricated antennas.

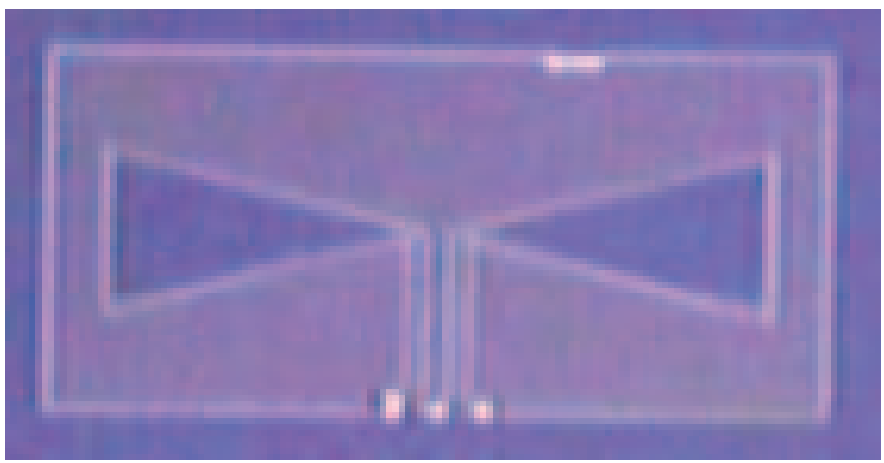

Fig. 2. The fabricated bow-tie antenna.

\section{Results and Discussion}

The antenna was measured using a J-band (220-325 GHz) Cascade Microtech millimeter wave vector network analyzer (VNA) with G-S-G infinity probes. The VNA was calibrated for 2-port S-parameter measurements using the short-openload-thru (SOLT) two port calibration process on a Cascade impedance substrate standard. Figure 3 shows the measured and simulated return loss of the designed bow-tie antenna. The bandwidth spans almost the entire frequency band from 220$325 \mathrm{GHz}$. This bandwidth is by far larger than that obtained from microstrip antennas. The discrepancy between the measured and simulated results can be attributed to unwanted signal reflections from surrounding objects during measurements.

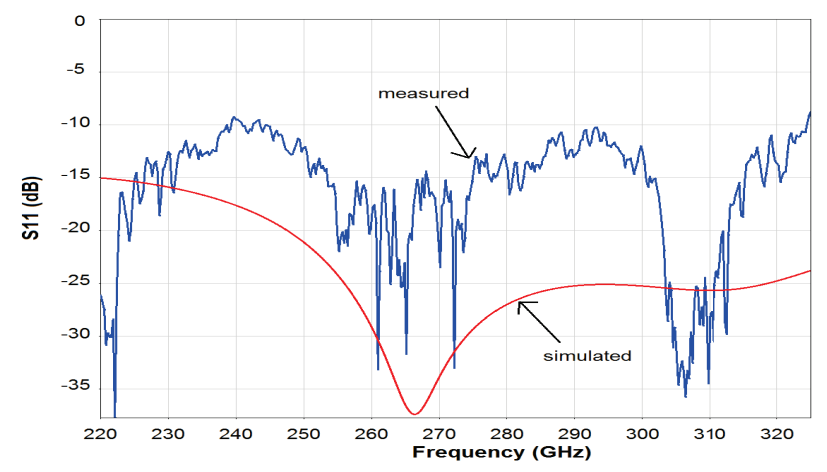

Fig. 3. Return loss of the bow-tie antenna on InP substrate.

The simulated radiation pattern is shown in Figure 4. Due to the large dielectric constant of the InP substrate, most of the radiation is directed towards the substrate. This is a common problem with antennas fabricated on high dielectric constant substrates and requires a practical solution in order to enable air-side radiation [11]. A practical solution to this problem has been to use a hemispherical lens on the backside to collect the power from the bottom side of the substrate [12-18]. Our current work (Section III) aims at designing antennas that can be integrated with RTD THz IC oscillators and which radiate into the air perpendicular to the substrate, to realise compact sources.

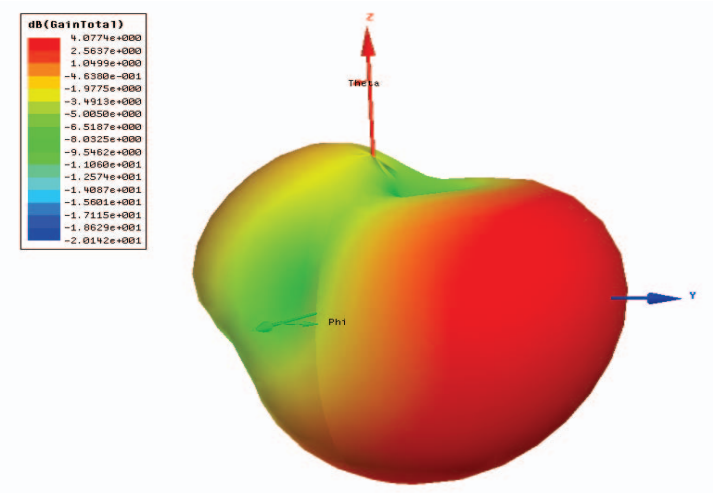

Fig. 4. Simulated radiation pattern of the bow-tie antenna on InP substrate at $300 \mathrm{GHz}$

\section{GROUNDED BOW-TIE ANTENNA ON THIN AND LOW DIELECTRIC CONSTATNT SUBSTRATE}

One possible way to achieve air side radiation is through employing a (thin) substrate of low dielectric constant and with a ground plane on the bottom side of the substrate to reflect the radiation upwards. The substrate used is benzocyclobutene (BCB) with dielectric constant $\epsilon_{r}=2.6$. This substrate is realised by spinning it, for instance, on a carrier substrate such as InP substrate with a coating of metallisation to act as the ground plane (and isolate the high permittivity InP substrate from the high frequency radiation). The antenna dimensions were calculated following the same procedure described in equations (1)-(4) with the only difference being that the substrate is grounded (ground plane is placed on the bottom side of the BCB substrate to act as a reflector). Therefore, the CPW 
dimensions ( $\mathrm{g}$ and $\mathrm{s}$ ) have to be calculated to get $50 \Omega$ characteristic impedance grounded-CPW line (GCPW). In initial investigations, the aim was to establish the thinnest possible $\mathrm{BCB}$ thickness that could provide acceptable performance and allow for simple fabrication processes. Through simulations, it was found that the antenna using a BCB thickness of 50 $\mu \mathrm{m}$ (designed for $300 \mathrm{GHz}$ ) gives optimal performance. The simulated return loss for the antenna is shown in Figure 5.

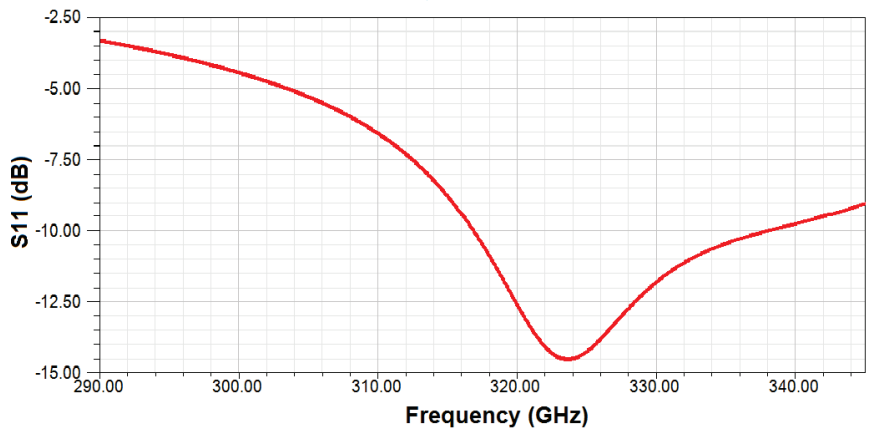

Fig. 5. Simulated return loss of the bow-tie antenna on $50 \mu \mathrm{m}$ thick grounded BCB substrate.

It can be seen that the antenna bandwidth extends from around $316 \mathrm{GHz}$ to $338 \mathrm{GHz}$ (for return loss less than $-10 \mathrm{~dB}$ ). The $3 \mathrm{D}$ radiation pattern of the antenna (at $321 \mathrm{GHz}$ ) is shown in Figure 6. The proposed antenna exhibits unidirectional radiation with single major lobe and radiates toward the air side (perpendicular to the substrate) which proves the advantages of both the low dielectric constant material and the ground plane placed on the bottom side of the BCB substrate. The frontto-back ratio (FBR) is about $12 \mathrm{~dB}$ and the beamwidth of the antenna is about $50^{\circ}$ in the xz-plane and $60^{\circ}$ in yz-plane. The antenna gain is $8.3 \mathrm{~dB}$ and the radiation efficiency is $95.6 \%$.

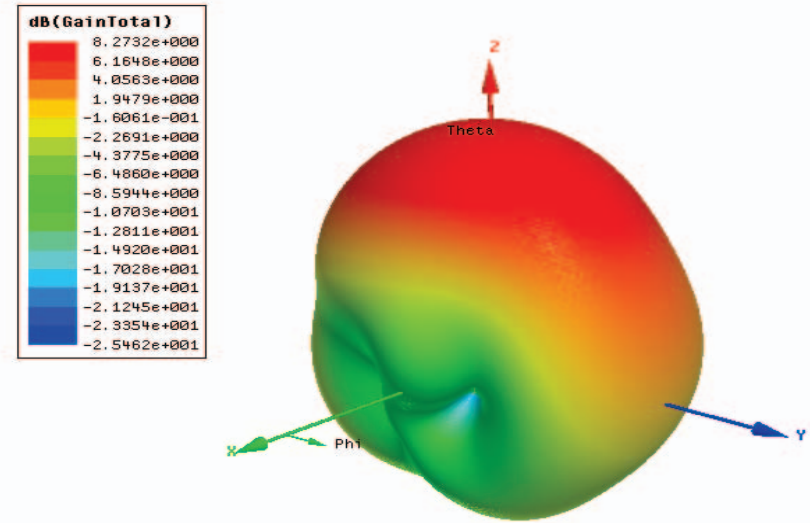

Fig. 6. Radiation pattern of the proposed bow-tie antenna on $50 \mu \mathrm{m}$ thick grounded BCB substrate at $321 \mathrm{GHz}$.

\section{EXPERIMENTAL VALIDATION OF THE PROPOSED CONCEPT}

Experimental validation of the proposed concept includes designing grounded bow-tie antenna using the Rogers substrate of thickness $762 \mu \mathrm{m}$ at lower frequency so the antenna can be measured in the anechoic chamber in the University of Glasgow which can measure radiation pattern of antennas up to around $20 \mathrm{GHz}$.
The first step is to determine the frequency of operation. In the previous design (bow tie on $50 \mu \mathrm{m} \mathrm{BCB}$ at $321 \mathrm{GHz}$ ) the $50 \mu \mathrm{m}$ is found to be:

$$
0.08 \lambda_{o} / \sqrt{\epsilon_{r}}=50 \mu m
$$

where $\epsilon_{r}=2.6$ (the dielectric constant of the BCB), and $\lambda_{o}$ is the free space wavelength at $321 \mathrm{GHz}$. Now, to determine the frequency for the $762 \mu \mathrm{m}$ Rogers $\left(\epsilon_{r}=3.48\right)$, we have:

$$
\begin{aligned}
& 0.08 \lambda_{o} / \sqrt{\epsilon_{r}}=762 \mu m \\
& \lambda_{o}=\frac{762 * 10^{-6} \sqrt{3.48}}{0.08}
\end{aligned}
$$

Thus, $f=17 G H z$.

The antenna was then designed for $17 \mathrm{GHz}$, simulated in HFSS and fabricated for experimental measurements. Figure 7 shows the return loss of the simulated and measured antennas. It can be seen that the simulation shows resonant at $17.7 \mathrm{GHz}$ (S11=-18 dB) while the measurement shows resonant at 16.5 $\mathrm{GHz}(\mathrm{S} 11=-28 \mathrm{~dB})$. Radiation patterns at $16.5 \mathrm{GHz}$ are shown in Figure 8 and Figure 9. Figure 10 illustrates the planes at which the patterns were plotted. Simulated and measured pattern in Figure 8 are in a good agreement with around 10 $\mathrm{dB}$ front-to-back ratio. The measured pattern in Figure 9 (in general) follows the simulated one (at many directions) although the ripples shown which might be caused by unwanted reflections from the SMA connector which is relatively large in size compared to the whole antenna geometry. Measured and simulated results show the feasibility of the proposed design. The proposed design does not require employment of bulky lenses attached to the substrate; the technique which has been widely used to extract the RF power and increase the bow-tie antenna gain [13-18].

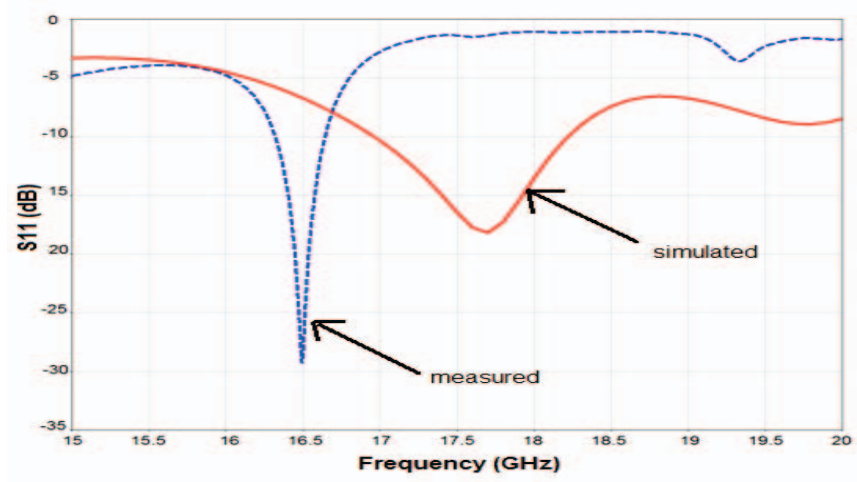

Fig. 7. Return loss of the bow-tie antenna on $762 \mathrm{~m}$ thick grounded Rogers substrate. (Red: Simulated. Blue: Measured)

\section{CONCLUSION}

In this paper, a grounded bow-tie slot antenna for terahertz applications was presented. It offers a known input impedance and radiates into the air perpendicular to the substrate. Future work is focused on integrating this antenna with InP-based RTD THz oscillators and designing the antenna so that most of the radiated energy is directed away from the semiconductor substrate. 


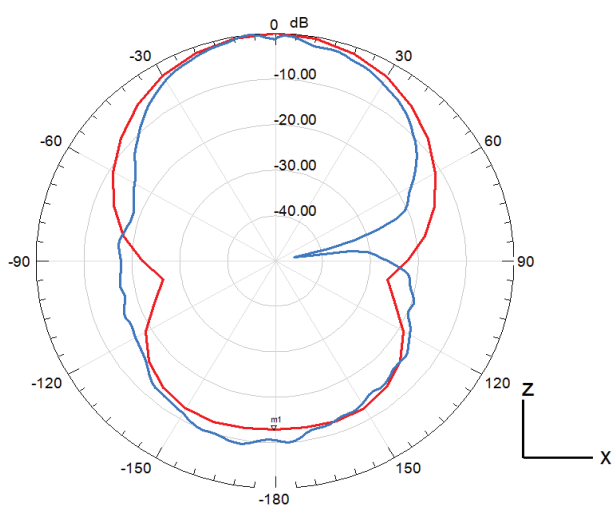

Fig. 8. Radiation pattern of antenna at $16.5 \mathrm{GHz}$ in zx-plane (Red: Simulation, Blue: Measurement).

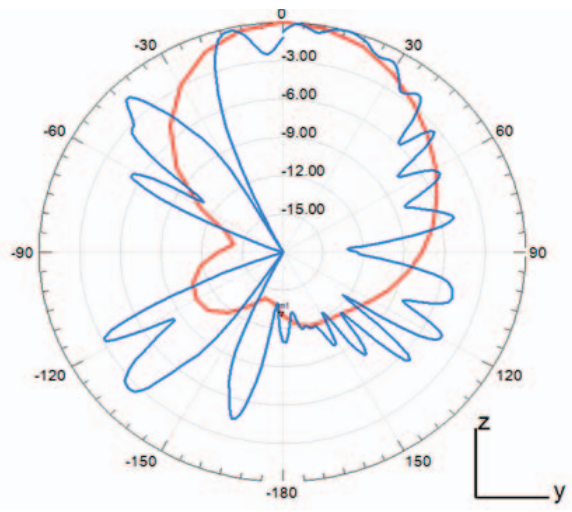

Fig. 9. Radiation pattern of antenna at $16.5 \mathrm{GHz}$ in yz-plane (Red: Simulation, Blue: Measurement).

\section{ACKNOWLEDGMENT}

This work was supported in part by the Engineering and Physical Sciences Research Council (EPSRC) under Grant EP/J019747/1

\section{REFERENCES}

[1] M. Tonouchi, "Cutting-edge terahertz technology," Nat. Photon, vol. 1, pp.97 -105 2007

[2] T. Kleine-Ostmann and T. Nagatsuma, "A Review on Terahertz Communications Research," J. Infrared, Millim. Terahertz Waves, vol. 32, pp.143 $-1712011$

[3] H. B. Liu , H. Zhong , N. Karpowicz, Y. Chen and X. C. Zhang, "Terahertz spectroscopy and imaging for defense and security applications,' Proc. IEEE, vol. 95, no. 8, pp.1514 -1527 2007

[4] M. Shiraishi , H. Shibayama , K. Ishigaki , S. Suzuki , M. Asada , H. Sugiyama and H. Yokoyama, "High output power $(\sim 400 \mu \mathrm{W})$ oscillators at around $550 \mathrm{GHz}$ using resonant tunneling diodes with graded emitters and thin barriers," Appl. Phys. Exp., vol. 4, pp.064101-1 -064101-3 2011

[5] M. Asada , S. Suzuki and N. Kishimoto, "Resonant tunneling diodes for sub-terahertz and terahertz oscillators," Jpn. J. Appl. Phys., vol. 47, no. 6, pp.4375 -4384 2008

[6] J. Wang, "Monolithic microwave/millimetrewave integrated circuit resonant tunnelling diode sources with around a milliwatt output power," Ph.D dissertation, Univ. Glasgow, 2014.

[7] K. Urayama, S. Suzuki, M. Asada, H. Sugiyama, and H. Yokoyama, "Sub-THz RTD oscillators integrated with planar horn antennas for horizontal radiation," In 33rd Int. Conf. on Infrared, Millimeter and Terahertz Waves (IRMMWTHz), Pasadena, California, US,15-19 Sept. 2008. pp. 1

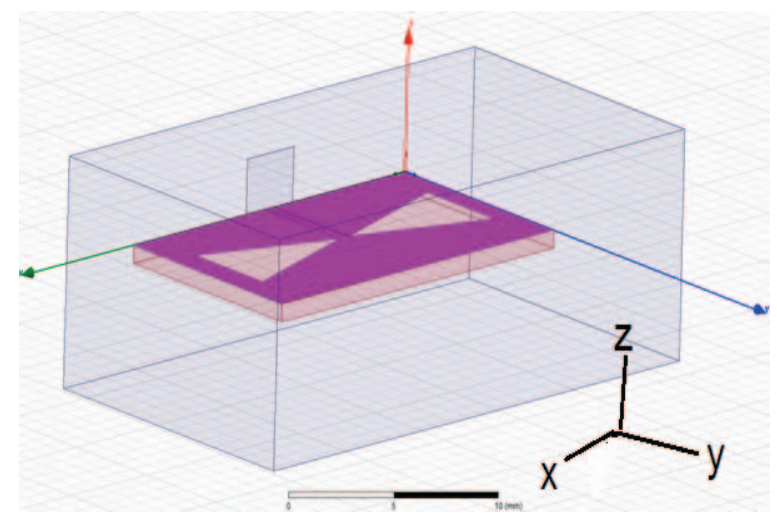

Fig. 10. Antenna geometry shows the planes at which the radiation patterns were plotted.

[8] K. Okada, S. Suzuki, and M. Asada, "Resonant-tunneling-diodeterahertz oscillator integrated with slot-coupled patch antenna," 26th Int. Conf. on Indium Phosphide and Related Materials (IPRM). Montpellier, 11th to15th May 2014. IEEE. pp. 1-2.

[9] A. S. Emhemmed and K. Elgaid, "Broadband micromachined microstrip patch antenna for G-band applications," European Microwave Conf. Rome, 2009. pp. 374-377.

[10] J. Wang, L. Wang, C. Li, B. Romeira, and E. Wasige, "28 GHz MMIC resonant tunnelling diode oscillator of around $1 \mathrm{~mW}$ output power," Electronics Letters, vol. 49, 2013, pp. 816-818.

[11] E. R. Brown, C. D. Parker and E. Yablonovitch, "Radiation properties of a planar antenna on a photonic-crystal substrate," J. Opt. Soc. Amer. B, Opt. Phys., vol. 10, pp.404 -410 1993

[12] N. Orihashi, S. Suzuki, and M. Asada, "One THz harmonic oscillation of resonant tunneling diodes," Appl. Phys. Lett., vol. 87, no. 23, pp.233501 2005.

[13] Y. Miyajima, T. Nukariya, and S. Suzuki, "Terahertz detector using 70-nm T-gate InAlAs/InGaAs HEMT integrated with bow-tie antenna," In 39rd Int. Conf. on Infrared, Millimeter and Terahertz Waves (IRMMWTHz), Tucson, AZ , 2014. pp. 1-2

[14] S. Boppel, M. Ragauskas, A. Hajo, M. Bauer, A. Lisauskas, S Chevtchenko, A. Ramer, I. Kasalynas, G. Valusis, J. Wurfl, W. Heinrich, G. Trankle, V. Krozer, and H. G. Roskos, "Terahertz edge detection with antenna-coupled field-effect transistors in $0.25 \mathrm{~m} \mathrm{AlGaN} / \mathrm{GaN}$ technology," In 39rd Int. Conf. on Infrared, Millimeter and Terahertz Waves (IRMMWTHz), Tucson, AZ, 2014. pp. 1.

[15] A. Mir, J. Yu, and X. Chen, "Design and Characterization of Cross bow tie Antenna Array for Focal plane array in Terahertz Imaging," In 39rd Int. Conf. on Infrared, Millimeter and Terahertz. Waves (IRMMWTHz), Tucson, AZ , 2014. pp. 1-2.

[16] C. R., Compton, R. C. McPhedran, Z. Popovic, G. M. Rebeiz, P. Tong, and D. B. Rutledge, "Bow-tie antennas on a dielectric half-space: theory and experiment," IEEE Trans. Antennas Propagat., vol. 35, pp.622 -631 1987

[17] R. Shireen, S. Shouyuan, and D. W. Prather, "Lens coupled bowtie antenna for millimeter wave operations," In IEEE Antennas and Propagat. Society Int. Symp., Honolulu, HI, 2007, pp.5055-5058.

[18] M. Weiss, A. G. Steffan, S. Fedderwitz, G. Tsianos, A. Stohr, and D. Jager, "Highly-compact fibre-optic package for $30-300 \mathrm{GHz}$ wireless transmitter modules," In 2nd Electronics System-Integration Technology Conference, Greenwich , 2008. pp.1111-1114.

[19] M. Koch, "Terahertz communications: A 2020 vision," In Terahertz Frequency Detection and Identification of Materials and Objects, pp. 325- 338, 2007.

[20] F. Lu, Q. Feng, and S. Li, "A novel CPW-fed bow-tie slot antenna for 5.8 GHz RFID tags," In Proc. PIERS, Cambridge, USA, 2008, pp.327 $-330$ 